\title{
BMJ Open Prevalence of multimorbidity in South Africa: a systematic review protocol
}

\author{
Rifqah A Roomaney (D) ,1,2 Brian van Wyk (D) ,2 Eunice Bolanle Turawa (D) ,1,3 \\ Victoria Pillay-van Wyk (D) ${ }^{1}$
}

To cite: Roomaney RA, van Wyk B, Turawa EB, et al. Prevalence of multimorbidity in South Africa: a systematic review protocol. BMJ Open 2020;10:e042889. doi:10.1136/ bmjopen-2020-042889

- Prepublication history and additional materials for this paper is available online. To view these files, please visit the journal online (http://dx.doi. org/10.1136/bmjopen-2020042889).

Received 18 July 2020 Revised 14 November 2020 Accepted 25 November 2020

D) Check for updates

(c) Author(s) (or their employer(s)) 2020. Re-use permitted under CC BY-NC. No commercial re-use. See rights and permissions. Published by BMJ.

${ }^{1}$ Burden of Disease Research Unit, South African Medical Research Council, Cape Town, South Africa

${ }^{2}$ School of Public Health, University of the Western Cape, Cape Town, South Africa

${ }^{3}$ Faculty of Medicine and Health Sciences, Community Health, Stellenbosch University, Cape Town, South Africa

Correspondence to Rifqah A Roomaney; rifqah.roomaney@mrc.ac.za

\section{ABSTRACT}

Introduction Multimorbidity has increased globally over the past two decades, due to ageing populations and increased burden of non-communicable diseases (NCDs). In a country like South Africa, with a growing burden of NCDs and a high prevalence of HIV, information on multimorbidity can improve planning for healthcare delivery and utilisation, and reduce costs in the context of constrained health resources. This review aims to synthesise prevalence studies on multimorbidity, and identify dominant clusters and trends of multimorbidity in South Africa.

Methods and analysis We will search electronic bibliographic databases (PubMed, Scopus, JSTOR, POPLINE, PsycINF0, ScienceDirect, Web of Science and CINAHL), and the reference lists of included articles. Two researchers will independently screen title and abstracts, and then full text to identify studies published before and in 2020 that report on prevalence of multimorbidity in South Africa. Risk of bias assessments will be done for each study. Information on the prevalence of multimorbidity and disease clusters will be extracted from each study. Where possible, prevalence of specific clusters of multimorbidity will be pooled using a random effects meta-analysis to account for variability between studies. The $\mathrm{I}^{2}$ statistic will be used to establish the extent of heterogeneity due to variation in prevalence estimates rather than due to chance. The systematic review will be reported according to the Preferred Reporting Items for Systematic reviews and Meta-Analyses.

Ethics and dissemination Only published journal articles will be included in the systematic review. This review received ethics approval as part of a larger project by the University of the Western Cape Biomedical Science Research Ethics Committee (BM20/5/8). The findings from this research will be used to estimate the prevalence of multimorbidity in South Africa and will contribute to the design of future research projects. The findings will be disseminated in a peer-reviewed journal article. PROSPERO registration number CRD42020196895.

\section{INTRODUCTION}

Multimorbidity, defined as the coexistence of two or more chronic conditions, has increased globally in the past two decades. ${ }^{12}$ Although a gold standard definition of multimorbidity has not been established, it has been recommended that the operationalisation of multimorbidity can include a combination of
Strengths and limitations of this study

To our knowledge, this will be the first systematic review to estimate the prevalence of multimorbidity in South Africa.

- This systematic review will include articles conducted in community/general and health-facility settings.

- This protocol follows the Preferred Reporting Items for Systematic reviews and Meta-Analyses Protocols $2015^{35}$ guidelines.

- The findings will be used to inform future research projects and can guide health policy and planning for service delivery in South Africa.

- The study may be limited by the way in which source articles define multimorbidity.

non-communicable diseases (NCDs), mental health conditions and infectious diseases. ${ }^{1}$ The increase in multimorbidity is attributed to ageing populations ${ }^{3}$ and the growing burden of NCDs. ${ }^{1}$ Whereas the increase in NCDs was initially predominantly observed in high-income countries; recently this increase was also reported in low and middle-income countries (LMICs). ${ }^{4}$

The prevalence of multimorbidity places additional stress on already severely strained health systems in LMICs $^{5}$ by driving up healthcare utilisation and costs. ${ }^{6-9}$ Furthermore, multimorbidity alters the patterns of individual health behaviours and access of health services; which in turn, has further implications for health systems responsiveness and pressing the urgency for further health reforms away from a programmatic approach to comprehensive, integrated services delivery. The need for reforms to integrate the treatment of various chronic conditions has been acknowledged. ${ }^{10}{ }^{11}$ Researchers have since highlighted the need to incorporate elements specific to people living with multimorbidity. These include the need for: multimorbid patients to have access to coordinated and multidisciplinary teams of health professionals; support for patients to self-manage their workload, and evidencebased guidelines applicable to multimorbid 
conditions. ${ }^{12-15}$ It is essential to grasp the magnitude and clustering of multimorbidity to inform health policy and regulations at a national level.

South Africa has a quadruple burden of disease of HIV/ AIDS, NCDs, injuries and other communicable diseases with perinatal conditions, maternal causes and nutritional deficiencies. ${ }^{1617}$ As HIV treatment has improved, people living with HIV in South Africa can expect a nearnormal life expectancy. ${ }^{18}$ However, ageing adults living with HIV are at a higher risk of developing chronic conditions such as NCDs compared with those not infected, and at an earlier age. ${ }^{19}$ An emerging pattern of HIV and NCD multimorbidity was described in the country. ${ }^{13}$ The burden of mental health conditions in people living with HIV has also been recognised ${ }^{20-23}$; with an estimated $40 \%$ of people living with HIV also afflicted by a diagnosable mental disorder. ${ }^{24} 25$ This demonstrates the complex burden of disease in the country.

Systematic reviews on multimorbidity that were conducted to date were mostly on older adults and in high-income countries. $^{26-30}$ These reviews reported pooled prevalences of multimorbidity between $38 \%$ and $66 \% .^{262831}$ In contrast, only two reviews included or were conducted on multimorbidity in LMICs. ${ }^{32}$ The 'global' systematic review included LMICs but limited the included studies to community settings; and did not include healthcare settings. ${ }^{31}$ Although the systematic review of multimorbidity in India and Bangladesh included primary healthcare settings, none were selected. ${ }^{33}$ The authors acknowledged that this was due to a gap in the multimorbidity literature in primary healthcare settings.

While community-based studies on multimorbidity are important, it is also essential for reviews on multimorbidity to include studies conducted in healthcare settings, because these studies can give a good indication of the number of people accessing healthcare for chronic conditions. ${ }^{29}$ Both community-based and health facility-based studies provide insight into the scale of the problem. To our knowledge, no study has been done to assess the prevalence of multimorbidity in South Africa that included studies in primary care settings. There is a need for research into multimorbidity in LMICs on the African continent as their disease burden differs from high income countries; that is, overlapping burdens of NCDs and infectious diseases which lead to new multimorbidity disease combinations. ${ }^{19}$ The proposed systematic review aims to synthesise existing literature on the prevalence of multimorbidity in South Africa, and identify common disease clusters and trends in the country.

\section{Research questions}

This systematic review aims to address the following review questions:

1. What is the prevalence of multimorbidity in persons over the age of 18 years in South Africa in studies reported up to and including 2020?

\begin{tabular}{|c|c|c|}
\hline Condition & Context & Population \\
\hline $\begin{array}{l}\text { Primary studies } \\
\text { reporting the } \\
\text { prevalence of } \\
\text { multimorbidity in } \\
\text { South Africa, using } \\
\text { acceptable case } \\
\text { definitions. }\end{array}$ & $\begin{array}{l}\text { South African } \\
\text { observational studies } \\
\text { up to and including } \\
2020 \text {. }\end{array}$ & $\begin{array}{l}\text { Males and } \\
\text { females, } \\
\text { aged } 18 \\
\text { years and } \\
\text { above } \\
\text { that reside } \\
\text { in South }\end{array}$ \\
\hline \multirow{2}{*}{$\begin{array}{l}\text { Studies focused on } \\
\text { 'multiple chronic } \\
\text { diseases', 'coexisting } \\
\text { conditions' and } \\
\text { 'multiple diseases' } \\
\text { will also be assessed } \\
\text { to determine if they } \\
\text { fit the definition of } \\
\text { multimorbidity. }\end{array}$} & $\begin{array}{l}\text { Study types include } \\
\text { population-based } \\
\text { studies and cross- } \\
\text { sectional studies. }\end{array}$ & Africa. \\
\hline & $\begin{array}{l}\text { Study settings include } \\
\text { ambulatory, community- } \\
\text { based, general practice, } \\
\text { primary healthcare } \\
\text { and other healthcare } \\
\text { settings. }\end{array}$ & \\
\hline
\end{tabular}

2. What is the prevalence of multimorbidity in adult males and females stratified by age group in South Africa in studies reported up to and including 2020?

3. What are common multimorbidity disease clusters in South Africa in studies reported up to and including 2020?

4. What are the trends in the prevalence of multimorbidity disease clusters in South Africa over time?

\section{METHODS AND ANALYSIS}

\section{Protocol and registration}

The methods for this systematic review was developed in accordance with the Preferred Reporting Items for Systematic Reviews and Meta-Analyses (PRISMA) ${ }^{34}$ and the PRISMA Protocols statement (online supplemental appendix 1). ${ }^{35}$ The systematic review is registered with the International Prospective Register of Systematic Reviews.

\section{Eligibility criteria}

The Condition, Context and Population (CoCoPop) mnemonic for prevalence systematic reviews ${ }^{36}$ will be used to define the inclusion criteria (table 1).

\section{Condition}

Articles about the prevalence of multimorbidity in South Africa will be included. Case definitions for multimorbidity may be defined in various ways. Ideally, articles will need to provide a clear description and definition of how they have conceptualised multimorbidity. For articles where multimorbidity has not been clearly defined, this article will adopt an operational definition of multimorbidity, similar to Pati, Swain. ${ }^{33}$ Their operational definition states 'studies documenting two or more chronic 
conditions, even though not mentioning the term multimorbidity'. ${ }^{34 p .4]}$ The article will also need to be explicit about which diseases have been included in their definition of multimorbidity.

The way in which multimorbidity was measured will need to be clearly defined. For example, whether a count of conditions was done. Also, the way in which diseases were diagnosed must be clear (eg, self-report, clinical assessment, laboratory test).

\section{Context}

Articles must report on studies conducted in South Africa. The setting of the study can be community-based, or health facility-based (eg, inpatient and outpatient).

\section{Population/types of participants}

This systematic review will be restricted to people who reside in South Africa and are over the age of 18 years old. Ideally, articles should report on the prevalence of multimorbidity by age group and sex.

\section{Outcomes}

The primary outcome will be the prevalence of multimorbidity in South Africa.

\section{Types of studies}

Observational, cross-sectional study designs are the most appropriate for determining the prevalence of a health condition, particularly common conditions of long duration. ${ }^{36}$ Thus, included articles will be cross-sectional or population-based study designs.

Articles must document the prevalence of multimorbidities, or their data must allow for the calculation of multimorbidity prevalence. Articles will need to define multimorbidity as the coexistence of at least two disease conditions or an operational definition will be applied. Articles with a sample size of less than 100 participants will be excluded as these studies may be susceptible to the influence of extreme observations. ${ }^{37}$ All articles before and including 2020 will be included. Additionally, in order to be included in this study, articles must be original and peer reviewed.

Inclusion and exclusion criteria are described in table 2. The following types of documents or studies will be excluded:

- Reviews, opinion pieces, conference presentations, letters, editorials, dissertations, abstracts, grey literature.

- Studies not conducted in South Africa.

- Studies that do not allow for the calculation of multimorbidity prevalence.

- Experimental studies.

- Studies not published in English.

\section{Search strategy}

A comprehensive search strategy was formulated by reviewing search terms used in other systematic reviews of multimorbidity. ${ }^{26-31} 33$ 38-41 The search strategy will be modified, where necessary, according to the database or
Table 2 Summary of inclusion and exclusion criteria

\begin{tabular}{ll}
\hline Inclusion criteria & Exclusion criteria \\
\hline Peer-reviewed articles. & $\begin{array}{l}\text { Non peer-reviewed articles and } \\
\text { grey literature. }\end{array}$ \\
$\begin{array}{l}\text { Articles with more than } \\
100 \text { participants. }\end{array}$ & $\begin{array}{l}\text { Articles with smaller sample } \\
\text { sizes (<100 participants). }\end{array}$ \\
$\begin{array}{l}\text { Articles about } \\
\text { multimorbidity in adults in } \\
\text { South Africa. }\end{array}$ & $\begin{array}{l}\text { Articles not about } \\
\text { multimorbidity, not about adults } \\
\text { or not conducted in South }\end{array}$ \\
Observational studies. & $\begin{array}{l}\text { Africa. } \\
\text { Experimental studies. }\end{array}$ \\
$\begin{array}{l}\text { Articles where it is } \\
\text { possible to calculate } \\
\text { the prevalence of } \\
\text { multimorbidity. }\end{array}$ & $\begin{array}{l}\text { Articles where it is not possible } \\
\text { to calculate the prevalence } \\
\text { of multimorbidity or to only } \\
\text { calculate multimorbidity in a } \\
\text { subset of people with a disease. }\end{array}$ \\
\hline
\end{tabular}

search engine used (table 3). Reference lists of included articles will be screened for relevant articles. The following electronic databases will be searched: PubMed, Scopus, JSTOR, POPLINE, PsycINFO, ScienceDirect, Web of Science and CINAHL.

Search terms will include 'multimorbidity' and linguistic variations such as 'multi-morbidity', 'multimorbidities', 'multi-morbidities', 'multi morbidity', 'multi morbidities', multiple morbidities', 'multiple-morbidities'. Terms such as 'multiple conditions', 'multiple diseases', 'multiple chronic diseases', 'multiple chronic conditions', 'multiple illnesses', 'multiple diagnoses', 'multipathology', 'chronic condition', 'chronic diseases' ${ }^{33}$ will also be included. These terms will be further restricted by location 'South Africa' and by method and study design 'prevalence, epidemiology, pattern'. This will be done by using the 'AND' and 'OR' Boolean operators where appropriate.

The term 'comorbidity' is now accepted to be distinct from multimorbidity. ${ }^{40}$ This study will exclude the search term 'comorbidity' as was done by another multimorbidity systematic review. ${ }^{31}$ However, if the search results include comorbidity studies that examine the prevalence of multimorbidity, the eligibility of these studies will be considered.

\section{Study selection and eligibility criteria}

One reviewer will conduct the literature search. Once the search is run, citations will be downloaded into a reference management system such as EndNote (EndNote X8, Thomson Reuters). Duplicates will be excluded using the EndNote deduplication function. Citations will be exported from EndNote into Rayyan. ${ }^{42}$

\section{Selection process}

Two reviewers (RAR and EBT) will independently screen the titles and abstracts of the search output to select potentially eligible studies using prespecified eligibility criteria. The web-based system, Rayyan, will be used to manage the screening process. Rayyan was designed by the Qatar 


\begin{tabular}{|c|c|}
\hline Search & Query \\
\hline \#4 & Search ((\#3 NOT (animals(mh] NOT humans(mh)))) \\
\hline \#3 & Search (\#1 AND \#2) \\
\hline \#2 & $\begin{array}{l}\text { Search (South Africa(mh] OR South Africa*(tiab] } \\
\text { OR RSA [tiab] OR Africa, Southern(mh:noexp)OR } \\
\text { Southern Africa(tiab)) }\end{array}$ \\
\hline \#1 & $\begin{array}{l}\text { Search (Multimorbidity OR multi-morbidity } \\
\text { OR multimorbidity(tiab] OR "multi morbidities" } \\
\text { OR multimorbidity(mh] OR multimorbidit* OR } \\
\text { multimorbid OR multimorbidities OR multi- } \\
\text { morbidit* OR "multiple morbidities" OR "multiple- } \\
\text { morbidit*" OR multidisease* OR multi-disease* OR } \\
\text { "multiple disorder" OR "multiple illness" }\end{array}$ \\
\hline
\end{tabular}

Foundation to expedite systematic reviews by semiautomating the initial screening of titles and abstracts. ${ }^{42}$ The full-text of potentially relevant articles will be retrieved and independently reviewed by two review authors (RAR and EBT) for eligibility.

Eligible citations will be uploaded into a web-based electronic system, the Burden of Disease Review Manager (BODRevMAN). This system was designed by the Burden of Disease Research Unit of the South African Medical Research Council to facilitate, systemise and manage the process of systematic review, the risk of bias assessment and provide a summary of data extracted ${ }^{37}$ BODRevMAN is tailored to the systematic review of observational studies. It facilitates the independent quality assessment and extraction of information from included full-text articles. Any disagreements will be resolved through discussion or another review author (VP-vW or BvW) will add input to enable a consensus. A PRISMA flow diagram will detail the study selection decisions made.

The researchers will not be blinded to the authors, titles and institutions of potentially eligible studies. Where there are multiple studies from the same dataset, the most appropriate data will be included. Where additional information is needed, the authors of the study will be contacted. One reviewer will email the corresponding author and a maximum of two attempts will be made, separated by one week.

\section{Quality assessment}

Two reviewers will independently assess study characteristics and the risk of bias of each study. The reviewers will use a modified checklist ${ }^{43}$ based on the Hoy et $a l^{44}$ risk of bias tool for population-based prevalence studies and the Newcastle-Ottawa Scale ${ }^{45}$ for assessing the quality of nonrandomised studies (online supplemental appendix 2). The tool has been described previously. ${ }^{37}{ }^{46}$ Results from the two researchers will be compared and differences will be discussed between them. Where consensus is not reached, a third reviewer (VP-vW) will be called on to arbitrate. Studies with a high risk of bias will be excluded and the reasons for their exclusion will be noted.

\section{Data extraction}

Two reviewers will independently extract data using BODRevMAN. The data extraction facilitated by BODRevMAN is in accordance with recommendations by Munn $e t a l^{86}$ for prevalence systematic reviews. The following data will be extracted:

- Citation details: authors, title, journal and year.

- Study details: study design, study setting (community or health facility), timeframe for data collection, geographical location and sample size.

- Case definition: how multimorbidity was defined and how disease conditions were measured.

- Participant characteristics: age, sex, urban/rural, socioeconomic characteristics.

- Description of main results: percentage prevalence of multimorbidity $(\mathrm{n} / \mathrm{N})$ and $95 \%$ CIs. Prevalence of conditions stratified by age and sex. Information on the most common disease clusters in the study sample.

In addition, the aims of the study, the method of data analysis used and any points of difference that may affect the interpretation of findings ${ }^{36}$ will be noted.

\section{Data synthesis}

The results will be presented in accordance with the PRISMA statement. Studies that are deemed to have a moderate or low risk of bias will be included in the analysis. Where appropriate, graphs will be used to display the information from included articles. The quantitative results will be summarised for individual studies and will include point estimates and interval estimates (eg, 95\% CIs). The prevalence data will be broken down by age, sex and disease clusters if the information is available. Differences and similarities between studies will be documented. Possible trends will also be noted.

If articles are amenable to meta-analysis, this will be done using Stata V.15 (College Station, Texas). A random effects meta-analysis will be used. Estimates will be pooled to obtain a summary estimate and $95 \%$ CI. A $\chi^{2}$ test will be used to assess statistical heterogeneity and the $\mathrm{I}^{2}$ statistic will be used to assess the degree of homogeneity. ${ }^{47} 48$ Where there are more than ten studies, publication bias will be assessed with a funnel plot and an egger test. ${ }^{49}$

Where sufficient data exist, subgroup analysis will be conducted based on multimorbidity case definition used, study setting (eg, community or health facility), age groups, sex and time periods. Ideally, multimorbidity prevalence will be estimated by age group and sex.

If it is not possible to do a meta-analysis, the findings from included articles will be presented in summary tables that will include the year of data collection, the study type and setting (community or health facility-based), location of study, the definition of multimorbidity used in each study, the diseases and number of diseases included in the study and how they were ascertained (eg, measured or self-reported).

\section{Patient and public involvement}

No patients will be involved directly in this study. 


\section{ETHICS AND DISSEMINATION}

This systematic review will be the first to review all available studies reporting on the prevalence of multimorbidity in South Africa. This study will provide estimates that will be valuable to health services planning. It will also provide much needed information on multimorbidity on the African continent and in LMICs in general.

The findings from this systematic review will be written up using the PRISMA guidelines. It will be disseminated through publication in a peer-reviewed journal article and/or conference proceedings.

This study is a secondary analysis of published studies. The data from published studies cannot be linked to individuals. This review received ethics approval as part of a larger project by the University of the Western Cape Biomedical Science Research Ethics Committee (BM20/5/8).

Contributors RAR, VP-vW, BvW conceptualised the study. RAR wrote the first draft of the protocol. VP-vW, BvW, EBT provided input for subsequent drafts. All authors gave approval for publication.

Funding The work reported herein was made possible through funding by the Burden of Disease Research Unit at the South African Medical Research Council. RAR is funded through the South African Medical Research Council through its Division of Research Capacity Development under the Internship Scholarship Programme from funding received from the South African National Treasury. The content hereof is the sole responsibility of the authors and does not necessarily represent the official views of the South African Medical Research Council or the funders. Grant number: NA.

Competing interests None declared.

Patient consent for publication Not required.

Provenance and peer review Not commissioned; externally peer reviewed.

Supplemental material This content has been supplied by the author(s). It has not been vetted by BMJ Publishing Group Limited (BMJ) and may not have been peer-reviewed. Any opinions or recommendations discussed are solely those of the author(s) and are not endorsed by BMJ. BMJ disclaims all liability and responsibility arising from any reliance placed on the content. Where the content includes any translated material, BMJ does not warrant the accuracy and reliability of the translations (including but not limited to local regulations, clinical guidelines, terminology, drug names and drug dosages), and is not responsible for any error and/or omissions arising from translation and adaptation or otherwise.

Open access This is an open access article distributed in accordance with the Creative Commons Attribution Non Commercial (CC BY-NC 4.0) license, which permits others to distribute, remix, adapt, build upon this work non-commercially, and license their derivative works on different terms, provided the original work is properly cited, appropriate credit is given, any changes made indicated, and the use is non-commercial. See: http://creativecommons.org/licenses/by-nc/4.0/.

\section{ORCID IDs}

Rifqah A Roomaney http://orcid.org/0000-0003-3267-8484

Brian van Wyk http://orcid.org/0000-0003-1032-1847

Eunice Bolanle Turawa http://orcid.org/0000-0003-3722-1547

Victoria Pillay-van Wyk http://orcid.org/0000-0001-8772-7197

\section{REFERENCES}

1 The Academy of Medical Sciences. Multimorbidity: a priority for global health research, 2018. Available: https://acmedsci.ac.uk/filedownload/82222577 [Accessed 20 Apr 2020].

2 WHO. Multimorbidity: technical series on safer primary care. Geneva: World Health Organization, 2016. http://apps.who.int/iris/bitstream/ 10665/252275/1/9789241511650-eng.pdf

3 WHO. World report on ageing and health [online]. Geneva: World Health Organization, 2015. https://www.who.int/ageing/events/worldreport-2015-launch/en/
4 Gouda HN, Charlson F, Sorsdahl K, et al. Burden of noncommunicable diseases in sub-Saharan Africa, 1990-2017: results from the global burden of disease study 2017. Lancet Glob Health 2019;7:e1375-87.

5 Mills A. Health care systems in low- and middle-income countries. $N$ Engl J Med 2014;370:552-7.

6 Barnett K, Mercer SW, Norbury M, et al. Epidemiology of multimorbidity and implications for health care, research, and medical education: a cross-sectional study. Lancet 2012;380:37-43.

7 Frølich A, Ghith N, Schiøtz M, et al. Multimorbidity, healthcare utilization and socioeconomic status: a register-based study in Denmark. PLoS One 2019;14:e0214183.

8 Sum G, Salisbury C, Koh GC-H, et al. Implications of multimorbidity patterns on health care utilisation and quality of life in middle-income countries: cross-sectional analysis. J Glob Health 2019;9:020413.

9 Lehnert T, Heider D, Leicht $\mathrm{H}$, et al. Review: health care utilization and costs of elderly persons with multiple chronic conditions. Med Care Res Rev 2011;68:387-420.

10 World Health Organization. Innovative care for chronic conditions: building blocks for actions: global report, 2002. Available: https:// www.who.int/chp/knowledge/publications/icccglobalreport.pdf [Accessed 5 Jun 2020].

11 Wagner EH, Austin BT, Von Korff M. Organizing care for patients with chronic illness. Milbank Q 1996;74:511-44.

12 Leijten FRM, Struckmann V, van Ginneken E, et al. The SELFIE framework for integrated care for multi-morbidity: development and description. Health Policy 2018;122:12-22.

13 Oni T, McGrath N, BeLue R, et al. Chronic diseases and multimorbidity--a conceptual modification to the WHO ICCC model for countries in health transition. BMC Public Health 2014;14:575.

14 Struckmann V, Leijten FRM, van Ginneken E, et al. Relevant models and elements of integrated care for multi-morbidity: results of a scoping review. Health Policy 2018;122:23-35.

15 Palmer K, Marengoni A, Forjaz MJ, et al. Multimorbidity care model: recommendations from the consensus meeting of the joint action on chronic diseases and promoting healthy ageing across the life cycle (JA-CHRODIS). Health Policy 2018;122:4-11.

16 Bradshaw D, Steyn K, Levitt NS, et al. Non-communicable diseases - A race against time. South African Medical Research Council, 2011. Available: http://www.health.uct.ac.za/usr/health/research/groupings/ cdia/downloads/MRC policy brief.pdf [Accessed 5 Jul 2020].

17 Pillay-van Wyk V, Msemburi W, Laubscher R, et al. Mortality trends and differentials in South Africa from 1997 to 2012: second National burden of disease study. Lancet Glob Health 2016;4:e642-53.

18 Johnson LF, Mossong J, Dorrington RE, et al. Life expectancies of South African adults starting antiretroviral treatment: collaborative analysis of cohort studies. PLoS Med 2013;10:e1001418.

19 Thienemann F, Ntusi NAB, Battegay E, et al. Multimorbidity and cardiovascular disease: a perspective on low- and middle-income countries. Cardiovasc Diagn Ther 2020;10:376-85.

20 Bongongo T, Tumbo J, Govender I. Depressive features among adult patients receiving antiretroviral therapy for HIV in Rustenburg district, SA. S Afr J Psychiatr 2013;19:4-34.

21 Nyirenda M, Chatterji S, Rochat T, et al. Prevalence and correlates of depression among HIV-infected and -affected older people in rural South Africa. J Affect Disord 2013;151:31-8.

22 Kagee A, Saal W, De Villiers L, et al. The prevalence of common mental disorders among South Africans seeking HIV testing. AIDS Behav 2017;21:1511-7.

23 Fairall L, Petersen I, Zani B, et al. Collaborative care for the detection and management of depression among adults receiving antiretroviral therapy in South Africa: study protocol for the cobalt randomised controlled trial. Trials 2018;19:193.

24 Pillay Y. State of mental health and illness in South Africa. S Afr J Psychol 2019;49:463-6.

25 South African College of Applied Psychology. The shocking state of mental health in South Africa in 2019, 2019. Available: https://www. sacap.edu.za/blog/management-leadership/mental-health-southafrica/ [Accessed 7 Jul 2020].

26 Ofori-Asenso R, Chin KL, Curtis AJ, et al. Recent patterns of multimorbidity among older adults in high-income countries. Popul Health Manag 2019;22:127-37.

27 Violan C, Foguet-Boreu Q, Flores-Mateo G, et al. Prevalence, determinants and patterns of multimorbidity in primary care: a systematic review of observational studies. PLoS One 2014;9:e102149.

28 Salive ME. Multimorbidity in older adults. Epidemiol Rev 2013;35:75-83.

29 Fortin M, Stewart M, Poitras M-E, et al. A systematic review of prevalence studies on multimorbidity: toward a more uniform methodology. Ann Fam Med 2012;10:142-51. 
30 Marengoni A, Angleman S, Melis R, et al. Aging with multimorbidity: a systematic review of the literature. Ageing Res Rev 2011;10:430-9.

31 Nguyen H, Manolova G, Daskalopoulou C, et al. Prevalence of multimorbidity in community settings: a systematic review and meta-analysis of observational studies. J Comorb 2019;9:2235042X1987093.

32 Xu X, Mishra GD, Jones M. Mapping the global research landscape and knowledge gaps on multimorbidity: a bibliometric study. J Glob Health 2017;7:010414.

33 Pati S, Swain S, Hussain MA, et al. Prevalence and outcomes of multimorbidity in South Asia: a systematic review. BMJ Open 2015;5:e007235.

34 Moher D, Liberati A, Tetzlaff J, et al. Preferred reporting items for systematic reviews and meta-analyses: the PRISMA statement. PLoS Med 2009;6:e1000097.

35 Shamseer L, Moher D, Clarke M, et al. Preferred reporting items for systematic review and meta-analysis protocols (PRISMA-P) 2015: elaboration and explanation. BMJ 2015;349:g7647.

36 Munn Z, Moola S, Lisy K. Chapter 5: systematic reviews of prevalence and incidence. In: The Joanna Briggs Institute, 2017. https://wiki.joannabriggs.org/display/MANUAL/JBI+Reviewer\%27s+ Manual

37 Wyk P-van V, Roomaney RA, Awotiwon OF, et al. Burden of disease review manager for systematic review of observational studies: technical report version 1. Cape Town: South African Medical Research Council, 2017.

38 France EF, Wyke S, Gunn JM, et al. Multimorbidity in primary care: a systematic review of prospective cohort studies. Br J Gen Pract 2012;62:e297-307.

39 Huntley AL, Johnson R, Purdy S, et al. Measures of multimorbidity and morbidity burden for use in primary care and community settings: a systematic review and guide. Ann Fam Med 2012;10:134-41.

40 Johnston MC, Crilly M, Black C, et al. Defining and measuring multimorbidity: a systematic review of systematic reviews. Eur $\mathrm{J}$ Public Health 2019;29:182-9.

41 Prados-Torres A, Calderón-Larrañaga A, Hancco-Saavedra J, et al. Multimorbidity patterns: a systematic review. J Clin Epidemiol 2014;67:254-66.

42 Ouzzani M, Hammady H, Fedorowicz Z, et al. Rayyan-a web and mobile APP for systematic reviews. Syst Rev 2016;5:210.

43 Wyk P-van V, Gbabe O, Roomaney R, eds. Risk of bias tool for systematic review of observational studies. Society for epidemiologic research 2015 Annual Meeting, 2015.

44 Hoy D, Brooks P, Woolf A, et al. Assessing risk of bias in prevalence studies: modification of an existing tool and evidence of interrater agreement. J Clin Epidemiol 2012;65:934-9.

45 Wells GA, Tugwell P, O'Connell D, et al. The Newcastle-Ottawa Scale (NOS) for assessing the quality of nonrandomized studies in meta-analyses [online], 2015. Available: http://www.ohri.ca/ programs/clinical_epidemiology/oxford.asp [Accessed 31 Jan 2020].

46 Pheiffer C, Pillay-van Wyk V, Joubert JD, et al. The prevalence of type 2 diabetes in South Africa: a systematic review protocol. BMJ Open 2018;8:e021029.

47 Higgins JPT, Thompson SG. Quantifying heterogeneity in a metaanalysis. Stat Med 2002;21:1539-58.

48 Higgins JPTet al. Measuring inconsistency in meta-analyses. BMJ 2003;327:557-60.

49 Egger M, Davey Smith G, Schneider M, et al. Bias in meta-analysis detected by a simple, graphical test. BMJ 1997;315:629-34. 